\title{
Sensor Selection Cost Function to Increase Network Lifetime with QoS Support
}

\author{
Ou Yang \\ Department of Electrical and Computer Engineering \\ University of Rochester \\ Rochester, NY, USA \\ oyang@ece.rochester.edu
}

\author{
Wendi B. Heinzelman \\ Department of Electrical and Computer Engineering \\ University of Rochester \\ Rochester, NY, USA \\ wheinzel@ece.rochester.edu
}

\begin{abstract}
Single-hop centralized wireless sensor networks are widely used for applications ranging from security and surveillance to medical monitoring. Often the goal of these networks is to provide satisfactory quality of service (QoS) to the application under different system states, but it is difficult to determine how the appropriate sensor sets should be selected over time, given the knowledge of all possible sensor sets to support the application QoS requirements. To address this problem, in this paper we propose a novel cost function called SUI (Sensor Usage Index) that is based on a sensor's relative ideal lifetime (SRIL) and can be used to select sensor sets so as to meet application QoS requirements for extended periods of time. Simulation results show that utilizing the proposed cost function for sensor set selection enables the network to meet application QoS requirements for longer than using other standard sensor selection schemes. In fact, our scheme approaches the optimal network lifetime, which can be found using global knowledge of the sensors and the system dynamics.
\end{abstract}

\section{Categories and Subject Descriptors}

C.2.1 [Computer-Communication Networks]: Network Architecture and Design - Network Communications, Wireless Communication; C.4 [Performance of Systems]: Design Studies

\section{General Terms}

Algorithms, Performance, Design

\section{Keywords}

Sensor selection; Cost function; QoS requirements

\section{INTRODUCTION}

Wireless senor networks have been gaining increasing prominence for practical deployments [7, 11], especially for applications that utilize single-hop centralized networks such as avalanche rescue [14], on-body health monitoring [12], environmental monitoring [13], and others. Several authors have shown that single-hop centralized networks can be more energyefficient than their multi-hop counterparts [1, 6, 16, 19].

Permission to make digital or hard copies of all or part of this work for personal or classroom use is granted without fee provided that copies are not made or distributed for profit or commercial advantage and that copies bear this notice and the full citation on the first page. To copy otherwise, or republish, to post on servers or to redistribute to lists, requires prior specific permission and/or a fee.

MSWiM’08, October 27-31, 2008, Vancouver, BC, Canada.

Copyright 2008 ACM 978-1-60558-235-1/08/10...\$5.00.
Meanwhile, single-hop centralized networks are cost-effective, easy to deploy and efficient in terms of scheduling data transmissions. Hence, in this paper we consider single-hop centralized wireless sensor networks, with the goal of increasing network lifetime with quality of service (QoS) support by proposing a new cost function.

For wireless sensor networks, prolonging the network lifetime with QoS support is of great importance, as the sensors are usually battery-powered, and hence highly constrained in terms of their available energy. However, previous sensor selection schemes $[3,4,15,17]$ are not optimal in terms of extending the network lifetime, as the cost functions they rely on do not simultaneously consider the diversity of various sensors in the network in terms of power consumption, remaining energy and contribution to the application QoS, and hence they cannot reflect a comprehensive estimate of a sensor's value to the application.

We therefore propose a novel cost function that can be used for sensor selection in single-hop centralized networks. Our cost function, Sensor Usage Index (SUI), is defined as a sensor's relative ideal lifetime divided by its actual remaining lifetime. Sensors that have a low SUI can be used more liberally than sensors that have a high SUI. Simulation results show that using SUI for sensor selection outperforms previous cost functions in both homogeneous networks and heterogeneous networks. In fact, networks that perform sensor selection based on SUI have lifetimes that approach the optimal network lifetime.

The rest of this paper is organized as follows. Section 2 discusses the motivation for our new cost function, and it provides discussion of related work in this area. Section 3 describes the proposed cost function, SUI, in detail. Section 4 analyzes our simulation results, and Section 5 concludes the paper.

\section{MOTIVATION AND BACKGROUND}

Wireless sensor networks oftentimes have redundancies, as multiple sensors can have overlapping contributions to the application's functionality. Hence, in a particular scenario, more than one sensor set may provide QoS support to an application. Much work [2, 5] has been done to determine all the possible sensor sets that satisfy the application QoS requirements. Given the knowledge of all those possible sensor sets, the challenge that arises is how a sensor selection scheme chooses one of these possible sensor sets as the final activated sensor set, so as to prolong the network lifetime with QoS support.

The simplest sensor selection scheme is to choose the sensor set randomly from among the sets that meet application QoS requirements. However, random selection cannot optimize the network lifetime by selectively using the sensors. Another scheme 
$[4,17]$ is to select the sensor set that consumes minimum power in each round of transmission. When more than one sensor set have the same total power consumption, the final sensor set is randomly selected from among those with minimum power. This scheme conserves energy for the whole network, but it might not optimize energy usage for individual sensors. Some important sensors used to support the application's functionality might die early if they happen to have small power consumption and low remaining energy. The third sensor selection scheme $[3,15]$ takes into account the idea that we should avoid selecting sensors with less remaining energy, if possible. Hence $1 / E$, where $E$ is a sensor's remaining energy, is proposed as the sensor's cost function. In this selection scheme, a smaller cost means that it is more preferable that the sensor be used. Hence the sensor set that has the minimum total cost among all possible sensor sets will be selected. However, the basic assumption behind the $1 / E$ scheme is that all the sensors in the network are homogeneous in power consumption. When this is not the case, a sensor's remaining lifetime depends on both its remaining energy as well as its power consumption. A sensor with large remaining energy might die soon if it has very large power consumption, and thus this sensor needs to be used conservatively.

In wireless sensor networks, sensors consume energy both in sensing data and in transmitting the sensed data to a base station. The power consumption for transmitting data is an exponential function of the distance from the sensor to the base station (assuming transmission power control is used), while the power consumption for sensing data is determined by the type of sensor (e.g., thermometer, pressure sensor, microphone, camera, etc.) as well as the sensing technology. Hence, different sensors may have very different power consumptions. As a result, for many applications that involve heterogeneous sensors (e.g., an avalanche rescue project [14] uses oximeters, oxygen sensors and accelerometers; a body sensor network [12] uses ECG sensors, $\mathrm{SpO} 2$ sensors, accelerometers, temperature and humidity sensors), the existing sensor selection schemes may not achieve desirable network lifetime, since neither the sensor's power consumption nor the sensor's remaining energy can be used individually to optimally prolong the network lifetime with QoS support. We therefore propose a cost function called SUI (Sensor Usage Index) that instead considers a sensor's remaining lifetime, which is a function of both the sensor's power consumption and the sensor's remaining energy. For a given application, each sensor's relative ideal lifetime is evaluated based on the knowledge of all the possible sensor sets that support the application QoS requirements in various system states. A sensor's relative ideal lifetime reflects how long the sensor should be alive in order to achieve maximum network lifetime. A sensor's SUI is its relative ideal lifetime divided by its actual remaining lifetime. Thus SUI provides an idea of how much the sensor is overused. When selecting the final sensor set, high cost sensors will be avoided, if possible.

\section{SENSOR SELECTION}

Our proposed sensor selection scheme first introduces a novel cost function, SUI, which describes how much the sensor is overused compared to its relative ideal lifetime, and then establishes a set of criteria based on the cost function to select the most preferable sensor set, aiming at achieving longer network lifetime.

\subsection{SUI cost function}

To maximize the network lifetime, we want to use the sensors ideally, so that every sensor can contribute all its energy in supporting the application QoS. To achieve this goal, both energy constraints and QoS requirements need to be considered. First of all, consider the scenario where there are no energy constraints. In this case, the base station can randomly select one sensor set from all possible sensor sets to meet the application QoS. Hence, over time, when the base station has made numerous random selections from the possible sensor sets, each sensor set will support the application for approximately the same amount of time. Consequently, the more often a sensor appears in the possible sensor sets, the longer it tends to be used to support the application QoS. However, if we consider energy constraints, sensors cannot be alive forever. The lifetime of a sensor is determined by its initial energy and its power consumption, and hence the sensor may not be able to support the application in the same way as it did in the scenario of no energy constraints.

Suppose we were able to "assign" a fixed amount of energy arbitrarily to each sensor in the network. Each sensor should receive an amount of energy proportional to the relative amount it required in the scenario with no energy constraints. This will ensure that the network can support application QoS for the entire time it is operational, and at the end of the network lifetime, all the energy in the network (the energy of each individual sensor) will be used, and no energy will be wasted. We call this the "ideal" scenario, and we call a sensor's lifetime in this scenario its "ideal lifetime."

In reality, however, we cannot assign energy to the sensors. They have a fixed initial energy and a fixed power consumption, which determines their active lifetime. Thus we need to determine how long the sensor's actual lifetime is relative to its "ideal lifetime." To do this, first we define a sensor's relative ideal lifetime (SRIL), and then we compare this relative ideal lifetime with the sensor's actual remaining lifetime.

To find a sensor's SRIL, we must determine the number of sets in which the sensor is active, and the probability of needing to use those sets. Suppose an application runs in $N_{s}$ system states, each of which has corresponding QoS requirements and a probability of occurrence $P(i), i=1 . . N_{s}$. For each system state $i$, there are $N_{p}(i)$ possible sensor sets, described as $F(i, j), i=1 . . N_{s}, j=1 . . N_{p}(i)$, that can provide the required QoS. Then, for each sensor $k$,

$$
\operatorname{SRIL}(k)=\sum_{i=1 . . N_{s}}\left(P(i) \cdot \sum_{k \in F(i, j): j=1 . . N_{p}(i)} \frac{1}{N_{p}(i)}\right) .
$$

This definition shows three rules to calculate a sensor's contribution to the application QoS. First, sensors in a given sensor set are supposed to work simultaneously for the same amount of time when that sensor set is chosen to support the application's QoS. Second, since all possible sensor sets under a specific system state equally satisfy the application's QoS, they are assumed to ideally support the system state for the same amount of time (note, however, that some sensor sets may actually be chosen more often according to our proposed cost function, which utilizes SRIL). Third, each system state has a certain probability of occurrence, which also influences a sensor's contribution in the ideal case. For example, consider a warehouse 
monitoring application that runs in two system states, “open” and "closed", with the probability of occurrence of 0.7 and 0.3 , respectively. Suppose there are 8 sensors, named $s_{1} \ldots s_{8}$, involved in the application, and there are 2 possible sensor sets, $s_{1} s_{3} s_{5} s_{7} s_{8}$ and $s_{2} s_{3} s_{5} s_{7} s_{8}$, that can support the QoS requirements in the "open" state and 5 possible sensor sets, $s_{3} s_{7} s_{8}, s_{4} s_{7} s_{8}, s_{5} s_{6} s_{7} s_{8}$, $s_{1} s_{3} s_{5}$, and $s_{2} s_{3} s_{5}$, that can support the QoS requirements in the "closed" state. Take $s_{7}$ for example. $s_{7}$ appears in 2 out of 2 possible sensor sets in the "open" state, which has the probability of occurrence of 0.7 , and 3 out of 5 possible sensor sets in the "closed" state, which has the probability of occurrence of 0.3 . Hence,

$\operatorname{SRIL}(7)=0.7 \cdot(1 / 2+1 / 2)+0.3 \cdot(1 / 5+1 / 5+1 / 5)=0.88$.

This mean $s_{7}$ has to be active during $70 \%$ of the network lifetime to support the "open" state, and be active during $0.18 \%$ of the network lifetime to support the "closed" state.

Set as a reference to ideally use the sensors and maximize the network lifetime, a sensor's SRIL is compared to its remaining lifetime (SRL) as a cost function, called sensor usage index (SUI) to reflect the extent to which the sensor is being used.

$$
\operatorname{SUI}(k)=\operatorname{SRIL}(k) / \operatorname{SRL}(k) .
$$

In the ideal case, every sensor has the same SUI, so that the sensors can contribute all of their energy to the application and maximize the network lifetime. In the practical case, however, sensors have non-ideal lifetime, and consequently are diverse in their SUIs. The sensor with the highest SUI in the network is the bottleneck to prolonging the network lifetime. The lower the SUI, the less the sensor is being used compared to its ideal usage. Hence, sensors with lower SUI are preferred to be used early, as their remaining lifetimes are longer than the bottleneck sensor and hence can support extra network lifetime until the bottleneck sensor has to be used. However, as sensors' remaining lifetimes become shorter and shorter, new bottlenecks will arise. Finally, all the sensors keep being used at the same pace, so that they are utilized to their maximum limit, thereby prolonging the network lifetime.

Note that the knowledge of each system state's probability of occurrence is used in the calculation of SUI. However, these probabilities may not be accurately evaluated before starting the application, or sometimes the probabilities may vary over time. We therefore evaluate these probabilities of occurrence on the fly by adaptively updating the values according to the current system state and past experience. The initial probabilities of occurrence can be set as rough evaluations or arbitrary values. Assume $T_{i}$, $i=1 . . N_{s}$ represents the time for which the application has been running in system state $i$. The probability of occurrence of system state $i$ is updated as follows:

$$
P(i)_{\text {current }}=0.8 P(i)_{\text {past }}+0.2 T_{i} / \sum_{j=1}^{N_{s}} T_{j} .
$$

\subsection{Choosing the optimal sensor set}

Since a sensor set usually includes multiple sensors, each of which might have different SUI values, a set of criteria is needed to evaluate every possible sensor set based on the SUI of the

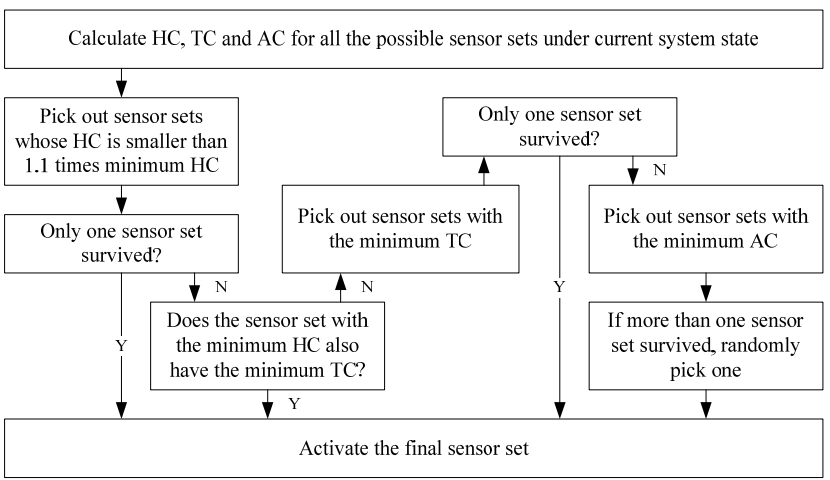

Figure 1. Sensor set selection.

individual sensors, so that the most preferable set is selected to prolong the network lifetime.

In each possible sensor set, the sensor with the highest cost, referred to as HC, determines the remaining lifetime of that sensor set. Thus, under a given system state, the sensor set with the lowest HC is preferred to be selected. This is the first selection criterion. The total cost of a sensor set, referred to as TC, tells how many resources are going to be used at one time. If two sensor sets have the same HC, the one with the smaller TC usually involves fewer sensors, and thus is preferred to be selected. However, if the $\mathrm{HC}$ of sensor set A is marginally smaller than the $\mathrm{HC}$ of sensor set $\mathrm{B}$, but sensor set $\mathrm{A}$ involves a large number of sensors leading to a large TC, while sensor set B uses only one sensor, thus having a very small TC, sensor set $\mathrm{B}$ is more preferable since a large gain on TC more than compensates for the tiny loss on $\mathrm{HC}$ in terms of prolonging the network lifetime. Hence, it is necessary to loosen the constraint on the lowest HC criterion. Thus, sensor sets with lower HC can also enter the second round selection based on TC. Finally, if two sensor sets have the same HC and TC, smaller average cost within the sensor set, referred to as AC, implies that all the sensors in that set have been used to a similar extent, and less energy will be wasted in the end. Thus, the sensor set with the lowest AC will be finally selected. Figure 1 shows the process of selecting sensor sets based on the individual sensors' costs.

\section{SIMULATION RESULTS}

We use MATLAB simulations to compare our proposed sensor selection scheme using SUI with random selection, minimum power selection and $1 / E$ selection, in terms of network lifetime with QoS support. In the simulations, system states are generated randomly according to their probabilities of occurrence, in units of 3 hours. The base station updates the activated sensor set and broadcasts a 100-byte beacon every half an hour to schedule a TDMA transmission accordingly. Activated sensors transmit data every 5 seconds with a 6-byte MAC header. We use different sensors with the same transceiver, CC2420 from Texas Instruments, an IEEE 802.15.4 radio. The power consumption parameters of the transceiver are listed in Table 1.

To examine the performance of the proposed sensor selection scheme, we discuss two different scenarios separately. One scenario considers homogeneous networks, where only one type of sensor is used. The other scenario considers heterogeneous networks, where multiple sensing variables are of interest to the 
Table 1. CC2420 transceiver parameters [18]

\begin{tabular}{|c|c|c|c|}
\hline Mode & Power $(\mathbf{m W})$ & Mode & Power $(\mathbf{m W})$ \\
\hline \multirow{5}{*}{ Transmit } & $25.5(<=18 \mathrm{~m})$ & & \\
& $29.7(<=56 \mathrm{~m})$ & Receive & 56.4 \\
& \begin{tabular}{c}
$33.0(<=100 \mathrm{~m})$ \\
\cline { 3 - 4 }
\end{tabular} & & \\
& $42.0(<=177 \mathrm{~m})$ & Sleep & 1.0 \\
\hline
\end{tabular}

Table 2. Sensors in warehouse monitoring system [8-10]

\begin{tabular}{|c|c|c|c|c|c|}
\hline Sensors & $\begin{array}{c}\text { Sensing } \\
\text { Power } \\
\text { (mW) }\end{array}$ & $\begin{array}{c}\text { Battery } \\
\text { Capacity } \\
\text { (mAH) }\end{array}$ & $\begin{array}{c}\text { Voltage } \\
\text { (V) }\end{array}$ & $\begin{array}{c}\text { Sensing } \\
\text { Radius } \\
\text { (m) }\end{array}$ & $\begin{array}{c}\text { Data } \\
\text { Rate } \\
\text { (bps) }\end{array}$ \\
\hline Vibration & 30 & 1200 & 9 & 3 & 24 \\
\hline Motion & 70 & 2000 & 3 & 4 & 240 \\
\hline Sound & 2.25 & 400 & 4.5 & 2 & 16 \\
\hline
\end{tabular}

application. Network lifetimes supported by each sensor selection scheme are compared to the optimal network lifetime, which is calculated by solving an optimization problem with the knowledge of each sensor's initial lifetime and the exact aposteriori probability of occurrence of each system state over the network lifetime. Practically, however, the exact probability of occurrence of each system state will vary over time and is hard to predict. Thus this method cannot be used in practice.

\subsection{Homogeneous networks}

In this scenario, 10 motion sensors, as specified in Table 2, are randomly located in a $10 \mathrm{~m} \times 10 \mathrm{~m}$ warehouse. For simplicity, they are assumed to have circular sensing areas with the specified radius. The warehouse monitoring system has two system states. System state 1 requires $100 \%$ coverage of the warehouse, while system state 2 requires no less than $80 \%$ coverage of the warehouse.

Figure 2 illustrates the network lifetime supported by random selection, minimum power selection, $1 / E$ selection and the proposed SUI selection compared with the optimal network lifetime, as system state 1's probability of occurrence increases from 0.1 to 0.9 . This figure shows that the proposed sensor selection scheme always achieves the longest network lifetime

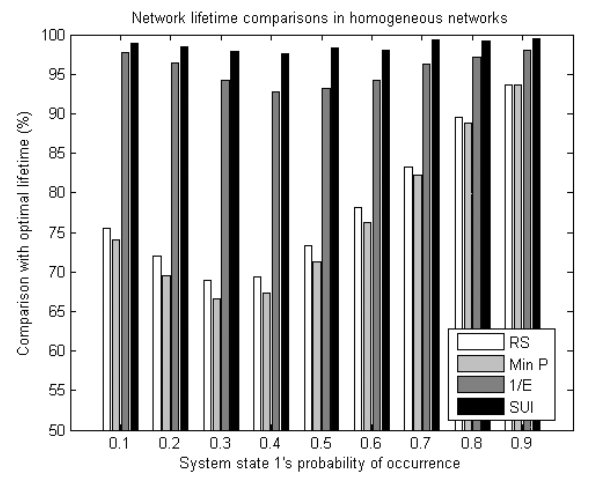

Figure 2. Network lifetime comparisons in homogeneous networks. among all the schemes, and it approaches the optimal network lifetime closely. 1/E selection also performs well, much better than random selection and minimum power selection. The proposed scheme and $1 / E$ selection have close performance because when the sensors are homogeneous, the proposed cost function for sensor $k$ is

$$
\operatorname{SUI}(k)=\frac{\operatorname{SRIL}(k)}{S R L(k)}=\frac{\operatorname{SRIL}(k) \times P}{E(k)} \propto \frac{\operatorname{SRIL}(k)}{E(k)}
$$

where $P$ is the same power consumption for every sensor, and $E(k)$ is the remaining energy of sensor $k$. Hence, losing the diversity of a sensor's power consumption, the proposed scheme can only take advantage of the sensor's SRIL to prolong the network lifetime compared with 1/E selection. On the other hand, minimum power selection does not use any diversity of a sensor's SRIL or remaining energy, so it performs even a bit worse than random selection. Another interesting phenomenon shown in Figure 2 is that the 4 sensor selection schemes achieve their best performances simultaneously when system state 1's probability of occurrence is 0.9. At this point, most of the time the application works in system state 1 , which requires $100 \%$ coverage of the warehouse and thus the redundancy in the network is low. Specifically, fewer possible sensor sets can support $100 \%$ coverage, and some sensors may be indispensable in all the possible sensor sets. Hence, the sensor selection scheme has fewer choices to improve the network lifetime, which is basically determined by these important sensors.

\subsection{Heterogeneous networks}

In the heterogeneous scenario, 3 motion sensors, 3 vibration sensors and 3 sound sensors, as specified in Table 2, are randomly located in a $10 \mathrm{~m} \times 10 \mathrm{~m}$ warehouse. Once again, they are assumed to have circular sensing areas with the specified radius. The application's QoS requirements remain the same as for the homogeneous network case, and again there are two system states: state 1 requires $100 \%$ coverage of the warehouse, while state 2 requires no less than $80 \%$ coverage of the warehouse.

Figure 3 compares the performance of random selection, minimum power selection, $1 / E$ selection and the proposed SUI selection scheme for heterogeneous networks. Even better performance is observed here than in the homogeneous scenario,

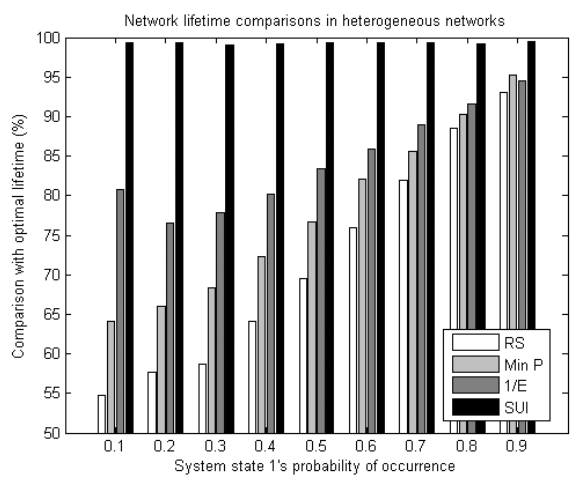

Figure 3. Network lifetime comparisons in heterogeneous networks. 
with the proposed sensor selection scheme achieving 99\% of the optimal network lifetime regardless of the system states' probabilities of occurrence. The other three sensor selection schemes, however, provide much shorter network lifetime. Generally, in heterogeneous networks, sensors have different power consumption, remaining energy and also SRIL, which reflects the sensor's contribution to the application's QoS. Neither minimum power selection nor 1/E selection incorporates even two of the three diversities. On the other hand, the proposed sensor selection scheme includes all of these factors into its cost function SUI, and hence always approaches the optimal network lifetime for both homogeneous and heterogeneous networks.

\section{CONCLUSIONS AND FUTURE WORK}

In this paper, we propose a sensor selection scheme that uses a cost function called SUI to prolong network lifetime with QoS support for single-hop centralized networks. SUI compares a sensor's relative ideal lifetime with its actual remaining lifetime. Simulation results show that the proposed cost function and the corresponding sensor selection criteria consider different diversities in a sensor's power consumption, remaining energy and QoS capability, hence outperforming the existing sensor selection schemes in both homogeneous networks and heterogeneous networks. Furthermore, the network lifetime supported by the proposed sensor selection scheme closely approaches the optimal network lifetime. Compared to existing sensor selection schemes, our approach has clear advantages, especially for heterogeneous, single-hop networks, as it extends network lifetime with QoS support.

Our future work will focus on shaping the proposed cost function for multi-hop distributed networks. First of all, routing needs to be considered by any cost function in multi-hop networks, and at the same time, SRIL must be evaluated without global knowledge of all the sensors, so that each sensor can independently make activation decisions.

\section{ACKNOWLEDGMENTS}

This work was supported in part by the National Science Foundation under grant CNS-0448046 and in part by a Young Investigator grant from the Office of Naval Research, \#N0001405-1-0626.

\section{REFERENCES}

[1] Bjornemo, E., Johansson, M., and Ahlen, A. 2007. Two hops is one too many in an energy-limited wireless sensor network. In Proceedings of IEEE International Conference on Acoustics, Speech and Signal Processing. ICASSP '07. 181-184.

[2] Cardei, M., Thai, M. T., Li, Y. and Wu, W. 2005. Energyefficient target coverage in wireless sensor networks. In Proceedings of $24^{\text {th }}$ Annual Joint Conference of the IEEE Computers and Communication Societies. INFOCOM '05. 1976-1984.

[3] Chang, J. and Tassiulas, L. 2000. Energy conserving routing in wireless ad hoc networks. In Proceedings of the $19^{\text {th }}$ Annual Joint Conference of IEEE Computers and Communications Societies. INFOCOM '00. 22-31.
[4] Chen, C., Tekinay, S., and Saraydar, C. 2004. Minimumpower \& energy-balancing cellular ad hoc augmented networks. In Proceedings of IEEE Wireless Communications and Networking Conference. WCNC '04. 1099-1103.

[5] Dasika, S., Vrudhula, S., Chopra, K., and Srinivasan, R. 2004. A framework for battery-aware sensor management. In Proceedings of Design, Automation and Test in Europe Conference and Exhibition. 962-967.

[6] Demirbas, M., Chow, K. Y., and Wan, C. S. 2006. INSIGHT: Internet-sensor integration for habitat monitoring. In Proceedings of International Symposium on a World of Wireless, Mobile and Multimedia Networks. WoWMoM '06.

[7] Hsieh, T. 2004. Using sensor networks for highway and traffic applications. IEEE Potentials. 23, 2 (April-May 2004), 13-16.

[8] http://www.digikey.com/scripts/dksearch/dksus.dll?Pname? Name=359-1001-ND\&site=us\&dkcid=10.

[9] http://www.isthq.com.

[10] http://www.ovt.com/data/parts/pdf/OV6680_PB(1.01)_web.p df.

[11] Kumagai, J. 2004. Life of birds [wireless sensor network for bird study]. IEEE Spectrum. 41, 4 (April 2004), 42-49.

[12] Lo, B. P. L., Thiemjarus, S., King, R. and Yang, G. 2005. Body sensor network - a wireless sensor platform for pervasive healthcare monitoring. Demonstrations in the $3^{\text {rd }}$ International Conference on Pervasive Computing. PERVASIVE '05.

[13] Martinez, K., Ong, R., Hart, J. K., and Stefanov, J. 2004. GLACSWEB: a sensor web for glaciers. In Adjunct Proceedings of $1^{\text {st }}$ European Workshop on Wireless Sensor Networks. EWSN '04. 56-62.

[14] Michahelles, F., Matter, P., Schmidt, A. and Schiele, B. 2003. Applying wearable sensors to avalanche rescue. Computers and Graphics. 27, 6 (Dec. 2003). 839-847.

[15] Perillo, M. and Heinzelman, W. 2004. DAPR: a protocol for wireless sensor networks utilizing an application-based routing cost. In Proceedings of IEEE Wireless Communications and Networking Conference. WCNC '04. 1540-1545.

[16] Schwieger K., and Fettweis, G. 2004. Multi-hop transmission: benefits and deficits. In Proceedings of the GI/ITG Fachgespräch "Sensornetze".

[17] Sharma, P., Narasimhan, A., Ramalingam, S., and Tripathi, S.K. 2006. Energy conservation in sensor networks through selective node activation. In Proceedings of International Symposium on a World of Wireless, Mobile and multimedia Networks. WOWMOM '06.

[18] Single-chip 2.4 GHz IEEE 802.15.4 Compliant and ZigBee(TM) Ready RF Transceiver - CC2420. http://focus.ti.com/docs/prod/folders/print/cc2420.html.

[19] Zhong, L. C., Rabaey, J. M., and Wolisz, A. 2005. Does proper coding make single hop wireless sensor networks reality: the power consumption perspective. In Proceedings of IEEE Wireless Communications and Networking Conference. WCNC '05. 664-669. 